\title{
Relationship between plasma cell-free DNA (cfDNA) and prognosis of TACE for primary hepatocellular carcinoma
}

\author{
Kun $\mathrm{Ma}^{1 *}$, Jiayun Liu ${ }^{2 *}$, Youjin Wang ${ }^{1}$, Yubin Zhong ${ }^{3}$, Zhenfeng $\mathrm{Wu}^{2}$, Ruiying $\mathrm{Fan}^{4}$, Shanfeng Guo ${ }^{1}$ \\ ${ }^{1}$ Department of Interventional Radiology, Jiangsu Province Hospital of Chinese Medicine, Affiliated Hospital of Nanjing University of Chinese \\ Medicine, Nanjing, China; ${ }^{2}$ Department of Surgical Oncology, Jiangsu Province Hospital of Chinese Medicine, Affiliated Hospital of Nanjing \\ University of Chinese Medicine, Nanjing, China; ${ }^{3}$ Department of General Surgery, Yixing People's Hospital, Yixing, China; ${ }^{4}$ Department of Medical \\ Oncology, Taikang Xianlin Drum Tower Hospital, Nanjing, China \\ Contributions: (I) Conception and design: S Guo; (II) Administrative support: None; (III) Provision of study materials or patients: None; (IV) \\ Collection and assembly of data: All authors; (V) Data analysis and interpretation: All authors; (VI) Manuscript writing: All authors; (VII) Final \\ approval of manuscript: All authors. \\ \#These authors contributed equally to this work as co-first authors. \\ Correspondence to: Shanfeng Guo. Department of Interventional Radiology, Jiangsu Province Hospital of Chinese Medicine, Affiliated Hospital of \\ Nanjing University of Chinese Medicine, 155 Hanzhong Road, Qinhuai District, Nanjing 210029, China. Email: wa27wa@aliyun.com.
}

Background: Our study aims to investigate changes in cell-free DNA (cfDNA) concentration and integrity in primary hepatocellular carcinoma (PHC) patients before and after transcatheter arterial chemoembolization (TACE) treatment and their influence on the evaluation of prognosis of the disease.

Methods: A total of 84 PHC patients admitted to the Affiliated Hospital of Nanjing University of Chinese Medicine from December 2016 to December 2017 were included as the study group, while 55 healthy people served as the control group. Plasma cfDNA concentration and integrity were determined using qRT-PCR. The correlation between cfDNA concentration/integrity and clinical characteristics of PHC patients were analyzed. A ROC curve was used to investigate the sensitivity and specificity of cfDNA as detection indices. Univariate and multivariate analyses were used to analyze factors affecting recurrence in PHC patients and compare recurrence-free survival (RFS) of PHC patients with high cfDNA expression and low cfDNA expression.

Results: Plasma cfDNA concentration and integrity were significantly higher in PHC patients before TACE treatment than in healthy people and significantly lower after treatment than before $(\mathrm{P}<0.05)$. The cfDNA concentration was significantly correlated with tumor size, lymph node metastasis, TNM stage, and BCLC stage, while cfDNA integrity was significantly correlated with tumor size, TNM stage, and BCLC stage $(\mathrm{P}<0.05)$. ROC results showed that the area under the curve $(\mathrm{AUC})$ value of cfDNA concentration was the largest, with an optimal cut-off of $10.51 \mathrm{ng} / \mathrm{mL}$. Multivariate regression analysis for COX showed that the TNM stage, cfDNA concentration, and AFP were independent risk factors that affected PHC patients' survival.

Conclusions: Plasma cfDNA concentration in PHC patients is more sensitive and specific than any other tumor marker. It is an independent risk factor for PHC patients treated with TACE. Therefore, it is hypothesized cfDNA is a potential biomarker for prognostic evaluation of PHC patients treated with TACE.

Keywords: Primary hepatocellular carcinoma (PHC); transcatheter arterial chemoembolization (TACE); cell-free DNA (cfDNA); independent risk factor; prognosis

Submitted Oct 14, 2020. Accepted for publication Dec 08, 2020.

doi: 10.21037/jgo-20-509

View this article at: http://dx.doi.org/10.21037/jgo-20-509

(C) Journal of Gastrointestinal Oncology. All rights reserved. 


\section{Introduction}

Primary hepatocellular carcinoma (PHC) is one of the most common malignant tumors. According to statistics, PHC ranks third in cancer-related mortality worldwide. On average, more than half a million people suffer from PHC each year $(1,2)$. Up till now, there are no specific targeted chemotherapy drugs for PHC. Moreover, PHC has a strong tolerance for radiotherapy and chemotherapy. Although surgical treatment is the most significant radical therapeutic strategy for PHC, not all patients meet the surgical conditions, especially patients with advanced PHC. There is also a high recurrence after resection $(3,4)$. However, liver transplantation is significant, but it takes time to find immune compatibility (5). With progress in treatment methods, PHC therapy has gradually moved from a single treatment model to a multi-disciplinary cooperative treatment mode, of which interventional therapy has become an essential auxiliary aspect.

Transcatheter arterial chemoembolization (TACE) is the preferred possibility for interventional treatment of liver cancer. PHC is a tumor with abundant blood supply, and it is supplied primarily by the hepatic artery. TACE is a highly selective injection of anticancer drugs into the arteries of liver tumors through a catheter to block the blood supply of the tumor. It is known that TACE blocks the hepatic artery and simultaneously releases a high concentration of chemotherapy drugs locally, resulting in ischemia, hypoxia, and necrosis of cancer tissues (6). The preferred adjuvant therapy for patients with PHC in early and advanced hepatocellular carcinoma, and those waiting for liver transplantation, is TACE, a consolidation therapy after surgical resection. Studies have shown that TACE significantly prolongs tumor-free survival and overall survival, whether as an adjunctive therapy before surgery (including liver tumor resection and liver transplantation) or as a preventive treatment after radical surgical resection $(7,8)$. Proper treatment is the key to TACE therapy. It has been reported in the literature that more than $90 \%$ of patients develop embolic syndrome after TACE. The duration and severity of embolic syndrome are closely related to the patient's progress and TACE treatment $(9,10)$. A study pointed out that the application of TACE alone as a local treatment of PHC can effectively control the tumors of $25 \%$ to $35 \%$ of patients and prolong the survival time of patients (11). There is a need to strictly control the number and interval of interventional treatment by accurately judging TACE's efficacy, but there is no unified standard for the efficacy evaluation of TACE for PHC at present. Therefore, there is a need to identify an appropriate biomarker for monitoring TACE's efficacy to avoid ineffective treatment, prevent unnecessary side effects, and change treatment regimens according to the disease's progress.

Cell-free DNA (cfDNA) is a free DNA fragment that exists in peripheral circulation, and it is produced during cell necrosis and apoptosis or in a small amount for selfrelease (12). The concentration of cfDNA in cancer patients is higher than that in healthy people. This phenomenon has long been known and has been repeatedly confirmed in many scientific studies (13). The level of cfDNA in cancer patients is affected by tumor stage, size, location, and other risk factors and prognostic factors. The effects of these changes vary from cancer to cancer. Many studies have shown that cfDNA integrity indicators can further improve cfDNA determination (14). The cfDNA fragments released from apoptotic cells have a uniform size through programmed enzyme hydrolysis, ranging from 185 to 200 bp. A larger proportion of cfDNA in plasma of tumor patients comes from necrotic cells, and the length and distribution of DNA fragments are different from those of healthy patients. Bioinformatics analysis can be used to estimate tumor load more accurately with concentration. Many researchers have investigated the prognostic value of cfDNA for tumor recurrence and patient survival in different cancers and its monitoring value for treatment response (15). Previously, a study pointed out that the level of cfDNA may be a potential indicator reflecting the prognosis of TACE. However, only 3 PHC patients were included in the study (16). The sample size was small and lacking convincing, and it did not include another potential indicator (cfDNA integrity). In this study, the concentration and fragment integrity of cfDNA in plasma before and after TACE treatment in $84 \mathrm{PHC}$ patients were determined to evaluate their potential as prognostic indicators for $\mathrm{PHC}$.

We present the following article in accordance with the REMARK reporting checklist (available at http://dx.doi. org/10.21037/jgo-20-509).

\section{Methods}

\section{Patient enrollment}

Eighty-four [84] patients with PHC who were scheduled for TACE treatment from December 2016 to December 2017 were selected as a study group. They formed 53 males and 31 females, aged $42-78$ years, with an average age of 
$58.13 \pm 4.65$ years. All procedures performed in this study involving human participants were in accordance with the Declaration of Helsinki (as revised in 2013). The Ethics Committee approved the study of the Affiliated Hospital of the Nanjing University of Chinese Medicine. All participants supplied signed informed consent.

Inclusion criteria: patients in the following categories were included: (I) patients who were clinically diagnosed according to the PHC diagnostic criteria proposed in the "Guidelines for the treatment of primary liver cancer (2017 edition)" issued by the Ministry of Health; (II) patients who were not on radiotherapy or chemotherapy, and (III) PHC patients who met the conditions for TACE. Exclusion criteria: (I) patients with coronary heart disease, cardiomyopathy, hematologic diseases, severe liver, kidney dysfunction, and acute cerebrovascular diseases; (II) patients who received ablation therapy, targeted drug therapy, and other treatments; (III) patients who already had liver metastases and whose survival was expected to be less than three months, and (IV) patients with tumors in more than $70 \%$ of the liver.

Also, 55 healthy people in our hospital's physical examination center during the same period were selected as the control group. They were made up of 37 males and 18 females, aged 33-68 years, with an average age of $56.16 \pm 4.43$ years.

\section{TACE treatment}

Each patient was placed in the supine position. After local anesthesia, the femoral artery was punctured and cannulated using the modified Seldinger method. Then, hepatic artery angiography was performed, and the tumor target artery was determined according to the angiographic results. The emulsion was prepared using $20-26.7 \mathrm{mg} / \mathrm{m}^{2}$ pirarubicin (Shenzhen Wanle Pharmaceutical Co. Ltd, H10930105), $85-100 \mathrm{mg} / \mathrm{m}^{2}$ oxaliplatin (Jiangsu Hengrui Pharmaceutical Co. Ltd, H20050962), $1.33-2.67 \mathrm{mg} / \mathrm{m}^{2}$ retetrexide (Nanjing Zhengda Tianqing Pharmaceutical Co. Ltd, H20090325), and 5-20 mL super liquid iodide oil (Guerbet, H20050307). Gelatin sponge granule (Hangzhou Alicang Medical Technology Co. Ltd, National Food and Drug Administration (quasi) word 2014 No. 3771056) were used to strengthen embolization after catheterization. The TACE treatment was performed every four weeks ( 3 times in all).

\section{Efficacy evaluation}

Enhancement examination and MR scan were performed one month after the end of treatment to evaluate short-term efficacy, with the modified Response Evaluation Criteria in Solid Tumors (mRECIST) viz: complete response (CR): all lesions completely disappeared; partial response (PR): diameter of the original lesion decreased by $>30 \%$; stable disease (SD): total diameter of lesions was not reduced to $\mathrm{PR}$ or increased to $\mathrm{PD}$; and progressive disease (PD): lesion diameter increased by $20 \%$ or new lesions appeared. The overall response $(\mathrm{ORR})=(\mathrm{CR}+\mathrm{PR}) /$ mean expanded. $\mathrm{A}$ follow-up of patients was conducted, and recurrence-free survival (RFS) in three patients was recorded.

\section{Determination of plasma cfDNA}

\section{Plasma sample collection and processing}

Three mL samples of venous blood from PHC patients (before and after TACE treatment) and healthy subjects were collected in EDTA-K2 anticoagulant tubes. After centrifugation at 3,000 rpm for $10 \mathrm{~min}$, each plasma sample was taken in a new $1.5 \mathrm{~mL}$ centrifuge tube centrifuged at low temperature and high speed for $10 \mathrm{~min}$ and stored at $-80^{\circ} \mathrm{C}$.

\section{Extraction of cfDNA from plasma}

QIAamp DNA Blood Mini Kit (Qiagen Germany, 51104) kit was used to extract cfDNA from plasma, and the specific operation was carried out in strict compliance with the kit instructions. The extracted DNA was determined in an ultraviolet spectrophotometer using the ratio of absorbances at 260 and $280 \mathrm{~nm}(\mathrm{~A} 260 / 280)$, and DNA samples with values 1.6-1.8 were taken. The DNA samples were either used for quantitative testing or stored in refrigerators at $-80^{\circ} \mathrm{C}$.

\section{Construction of the standard curve}

Human genomic DNA was subjected to gradient dilution to obtain seven standard s concentrations (1.0 to $0.0002 \mathrm{ng} / \mathrm{mL}$ ), which were used to construct a standard curve.

\section{qRT-PCR}

Repeat sequences of 97 and 300 bp DNA fragments were amplified. Ninty-seven bp primer characterized plasma cfDNA concentration, while the ratio of 97 to 300 bp indicated cfDNA integrity. The qRT-PCR system $(25 \mu \mathrm{L})$ comprised $2 \mu \mathrm{L}$ plasma DNA template, $12.5 \mu \mathrm{L}$ SYBR GREEN MASTER MIX (2×), $0.5 \mu \mathrm{L}$ upstream primer, $0.5 \mu \mathrm{L}$ reverse primer, $0.25 \mu \mathrm{L}$ TaqMan Prode, and $8.75 \mu \mathrm{L}$ double distilled water. The conditions were: pre-denaturation at $95^{\circ} \mathrm{C}$ for $1 \mathrm{~min}$; degeneration 
at $95{ }^{\circ} \mathrm{C}$ for $8 \mathrm{sec}$; annealing/extension at $60{ }^{\circ} \mathrm{C}$ for 15 sec; 35 cycles. After the cycle is over, the CT value is substituted into the standard curve to calculate the concentration of cfDNA.cfDNA integrity is the ratio of 300 to $97 \mathrm{bp}$ content. Primer 1 (97 bp): forward: 5 ' -TGGCACATATACACCATGGAA-3 ', reverse: 5'-TGAGAATGATGGTTTC-3', primer 2 (300 bp): forward: 5'-ACAACCTATTCCAAAATTGACCAC-3', reverse: 5'-TTCCCTCTACACACTGCTTTGA-3', the cfDNA integrity index is calculated as the ratio of LINE $300 \mathrm{bp}$ and LINE 197 bp qRT-PCR results. The internal reference b-action amplified fragment is $186 \mathrm{bp}$ : b-action upstream primer: 5'-TGGCACCCAGCACAATGAA-3', b-action downstream primer: 5'-CTAAGTCATAGTCCG CCTAGAAGCA-3'.

\section{Enzyme-linked immunosorbent assay (ELISA)}

The contents of alpha fetoprotein (AFP), carcinoembryonic antigen (CEA), carbohydrate antigen 199 (CA199), and alanine aminotransferase (ALT) in the plasma of the study group and control group were determined according to the instructions in their respective assay kits. The reference ranges are: $\mathrm{AFP} \leq 7 \mathrm{ng} / \mathrm{mL}, \mathrm{CEA} \leq 3.5 \mathrm{ng} / \mathrm{mL}$, CA199 $\leq 39 \mathrm{U} / \mathrm{mL}$, and $5.0 \mathrm{U} / \mathrm{L} \leq \mathrm{ALT} \leq 9.0 \mathrm{U} / \mathrm{L}$.

\section{Statistical analysis}

Measurement data are expressed as mean $\pm \mathrm{SD}$, and $t$-test or one-way ANOVA was used for statistical analysis. Counting data are expressed as numbers, and $\chi^{2}$ test is used for analysis. The specificity and sensitivity of different tumor markers were set up using ROC curves, and the cut-off value of cfDNA concentration was determined according to the ROC Youden index. Univariate analysis, survival analysis, and survival curve drawing were conducted using the KaplanMeier method. Differences in survival curves between distinct groups were determined with the log-rank test, while COX regression analysis was used for multivariate analysis. All statistical analyses were done with SPSS 21.0 software. Values of $\mathrm{P}<0.05$ were considered statistically significant.

\section{Results}

\section{Short-term efficacy TACE treatment in PHC patients}

Figure $1 A$ and $1 \mathrm{~B}$ are in line with the typical characteristics of "liver cancer". Before TACE, the right lobe of the liver occupies $7.62 \mathrm{~cm} \times 5.14 \mathrm{~cm}$, and the MR arterial phase shows obvious enhancement and the blood supply is abundant (Figure 1A). Before TACE, the MR portal vein phase shows the rapid disappearance of the contrast agent in the lesion (Figure 1B). 3.5 months after TACE, the lesion was significantly smaller than before $(5.15 \mathrm{~cm} \times 3.91 \mathrm{~cm})$, and the MR arterial phase showed complete disappearance of tumor enhancement (Figure 1C). 3.5 months after TACE, the MR portal phase showed a clear outline of the lesion and the tumor was completely inactivated (Figure 1D).

In the $84 \mathrm{PHC}$ patients treated with $\mathrm{EOX}$, there were 7 cases of CR, 46 cases of PR, 24 cases of SD, 7 cases of PD, with an ORR of $63.10 \%$.

\section{Plasma cfDNA concentration and integrity in bealtby people and PHC patients before and after TACE treatment}

Plasma cfDNA concentration was $5.93 \pm 2.11 \mathrm{ng} / \mathrm{mL}$ in the healthy control group, $19.76 \pm 2.68 \mathrm{ng} / \mathrm{mL}$ in $\mathrm{PHC}$ patients before TACE treatment $12.39 \pm 3.27 \mathrm{ng} / \mathrm{mL}$ in PHC patients after TACE treatment (Figure 2A). Plasma cfDNA integrity was $0.72 \pm 0.58$ in the healthy control group, $5.35 \pm 0.97$ in

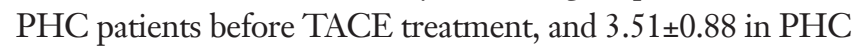
patients after TACE treatment (Figure $2 B$ ). Plasma cfDNA concentration was $11.04 \pm 3.68 \mathrm{ng} / \mathrm{mL}$ in the CR patients, $11.85 \pm 3.72 \mathrm{ng} / \mathrm{mL}$ in PR patients, $12.31 \pm 3.54 \mathrm{ng} / \mathrm{mL}$ in $\mathrm{SD}$ patients and $12.94 \pm 3.17 \mathrm{ng} / \mathrm{mL}$ in $\mathrm{PD}$ patients (Figure $2 C$ ). Plasma cfDNA integrity was $2.75 \pm 0.89$ in the CR patients, $3.36 \pm 0.92$ in $\mathrm{PR}$ patients, $4.27 \pm 0.95$ in $\mathrm{SD}$ patients, and 5.16 \pm 0.96 in PD patients (Figure 2D).

As shown in the results, plasma cfDNA concentration and PHC patients' integrity before TACE treatment were significantly higher than corresponding values for healthy people $(\mathrm{P}<0.05)$. After TACE treatment, plasma cfDNA concentration and integrity were significantly decreased compared with those before treatment and were negatively correlated with patient efficacy.

\section{The relationship between plasma cfDNA concentration and integrity with the clinical characteristics of PHC patients}

The correlation between plasma cfDNA concentration and integrity with the clinical characteristics before and after TACE treatment in 84 PHC patients were analyzed. The results showed that cfDNA concentration and $\mathrm{PHC}$ patients' integrity before and after TACE treatment were not correlated with age, gender, tumor size, differentiation degree, CEA, CA199, and ALT (P>0.05). However, before and after TACE treatment, cfDNA concentration 
A

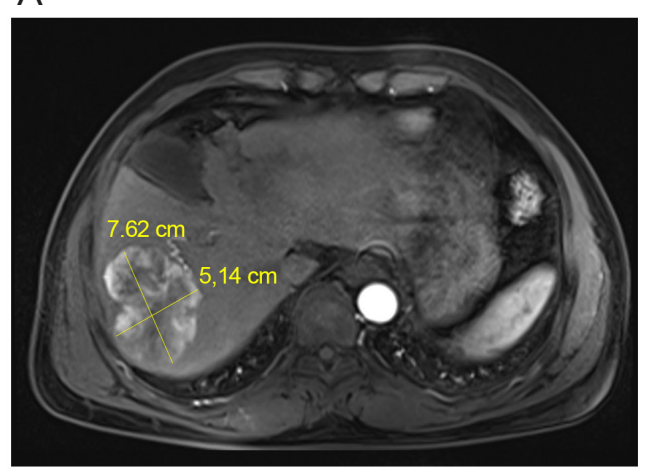

c

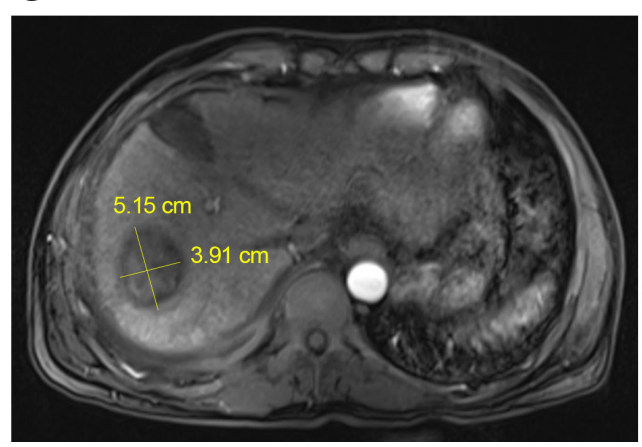

B

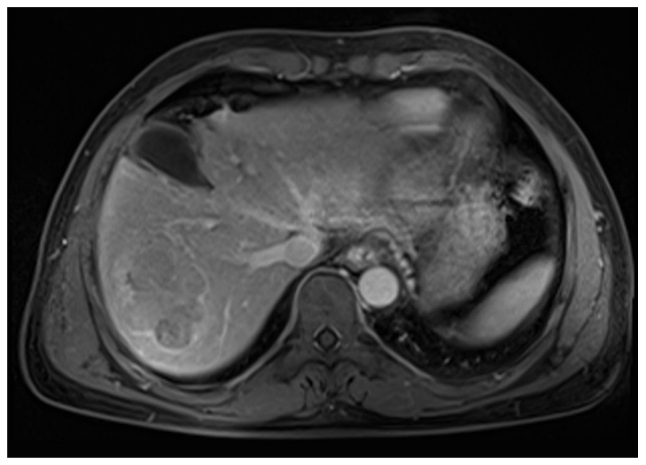

D

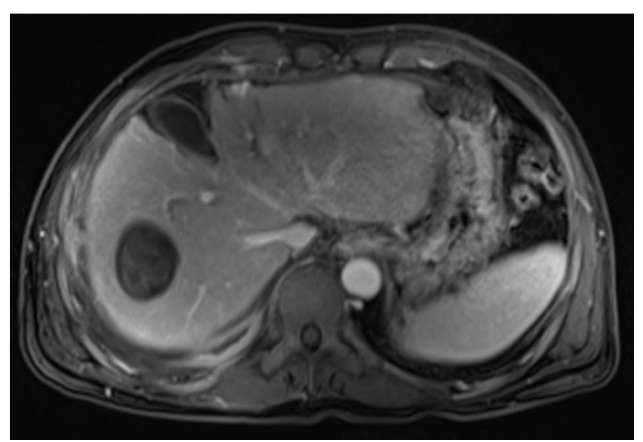

Figure 1 Representative images of MR before TACE and after TACE. (A) The right lobe of the liver was occupied by $7.62 \mathrm{~cm} \times 5.14 \mathrm{~cm}$ before TACE. MR artery showed obvious enhancement and rich blood supply; (B) MR portal vein stage before TACE showed rapid dissolution of contrast agent in lesions; (C) three and a half months after TACE, the lesion was significantly smaller (5.15 cm $\times 3.91 \mathrm{~cm}$ ) than that before TACE. MR arterial phase showed that tumor enhancement had completely disappeared; (D) three and a half months after TACE, MR portal vein stage showed clear outline of the lesion and complete inactivation of the tumor.

was significantly correlated with tumor size, lymph node metastasis, TNM stage, and BCLC stage, while cfDNA integrity was significantly correlated with tumor size, TNM stage, and BCLC stage $(\mathrm{P}<0.05)$. These results are presented in Tables 1 and 2.

\section{ROC curve}

The ROC curves of cfDNA concentration, cfDNA integrity, AFP, CEA, CA199, and ALT were set up, as shown in Figure 3. The area under the curve (AUC) of cfDNA concentration was 0.8775 (95\% CI: 0.7627-0.9923), the AUC of cfDNA integrity was 0.7625 (95\% CI: $0.6142-0.9108$ ); the AUC of AFP was 0.8304 (95\% CI: $0.6972-0.9628)$, the AUC of CA199 was 0.7204 (95\% CI: 0.5666-0.8834); the AUC of ALT was 0.7010 (95\% CI: $0.4567-0.8083$ ), and the AUC of CEA was 0.7223 (95\% CI: 0.5628-0.8833). The AUC of cfDNA concentration was significantly higher than AFP, CA199, ALT, or CEA, while cfDNA integrity was significantly higher than CA199, ALT, or CEA. The optimal cut-off value of cfDNA concentration was $10.51 \mathrm{ng} / \mathrm{mL}$ (sensitivity: $63.5 \%$, specificity: 91.2\%).

\section{Analysis of RFS after TACE treatment in PHC patients}

The PHC patients were followed up for one-and-half years (median follow-up time was 11.2 months). Two patients did not complete follow-up due to loss of contact. The RFS of the patients with cfDNA low expression was $9.26 \%$, which was significantly lower than cfDNA high expression patients whose RFS was $26.67 \%(\mathrm{P}=0.048)$. These results are shown in Figure 4 .

\section{Univariate and multivariate analyses of factors that affect survival after TACE treatment in PHC patients}

Kaplan-Meier survival analysis was performed for 84 PHC 

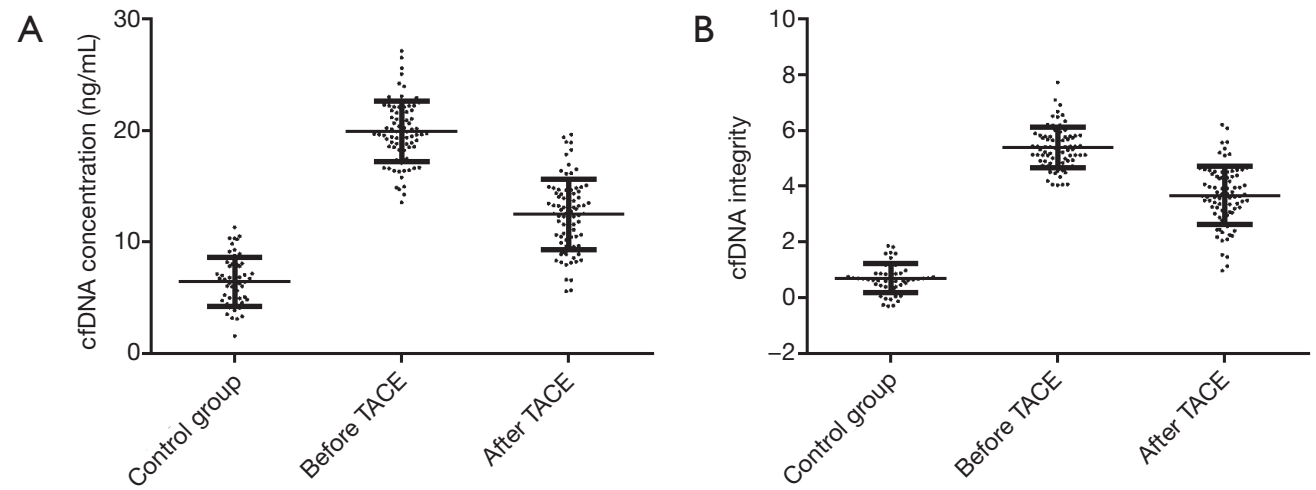

C

\section{D}
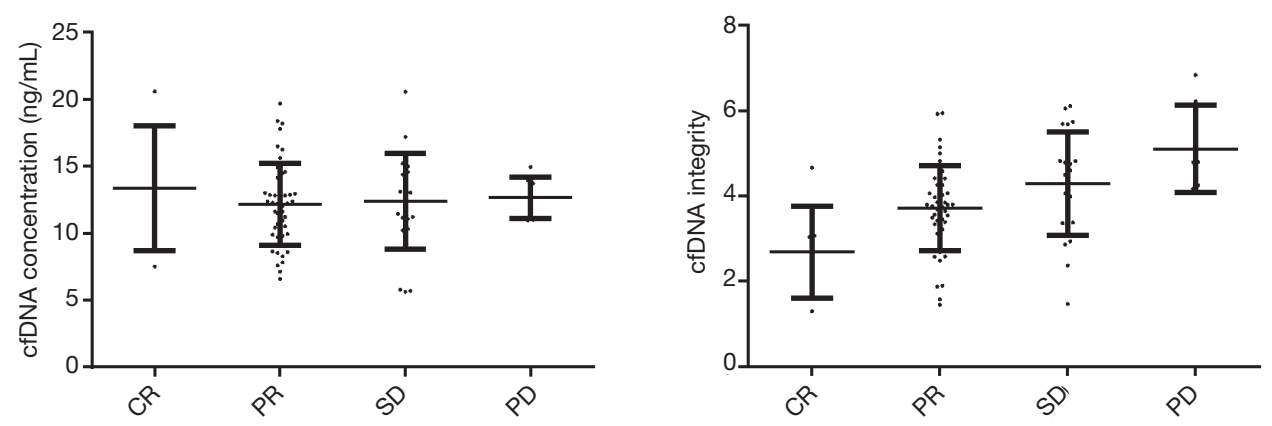

Figure 2 cfDNA concentration and integrity in healthy people and $\mathrm{PHC}$ patients. ${ }^{*} \mathrm{P}<0.05$ vs. control group; ${ }^{\#} \mathrm{P}<0.05$, compared with value before TACE treatment. cfDNA, cell-free DNA; PHC, primary hepatocellular carcinoma; TACE, transcatheter arterial chemoembolization.

Table 1 Relationship between cfDNA concentration and clinical characteristics of PHC patients

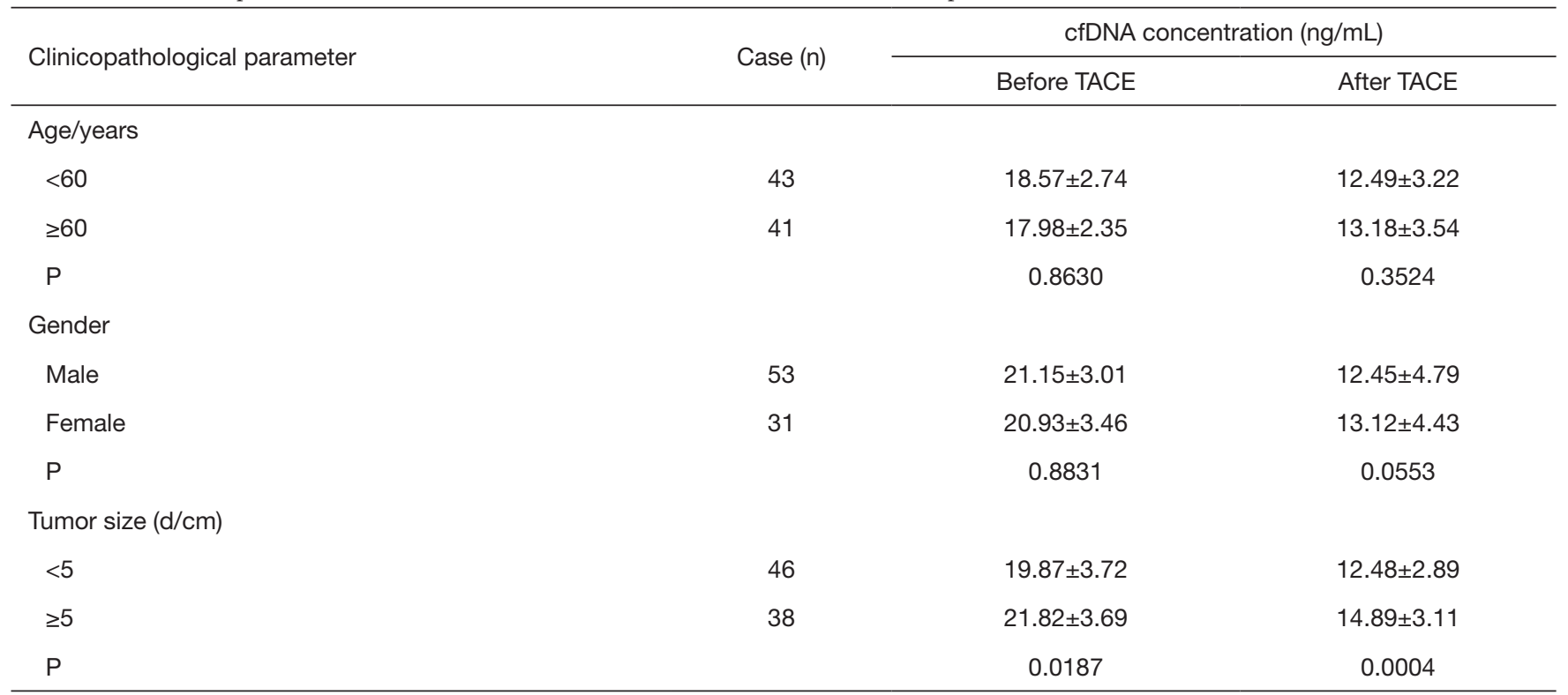

Table 1 (continued) 
Table 1 (continued)

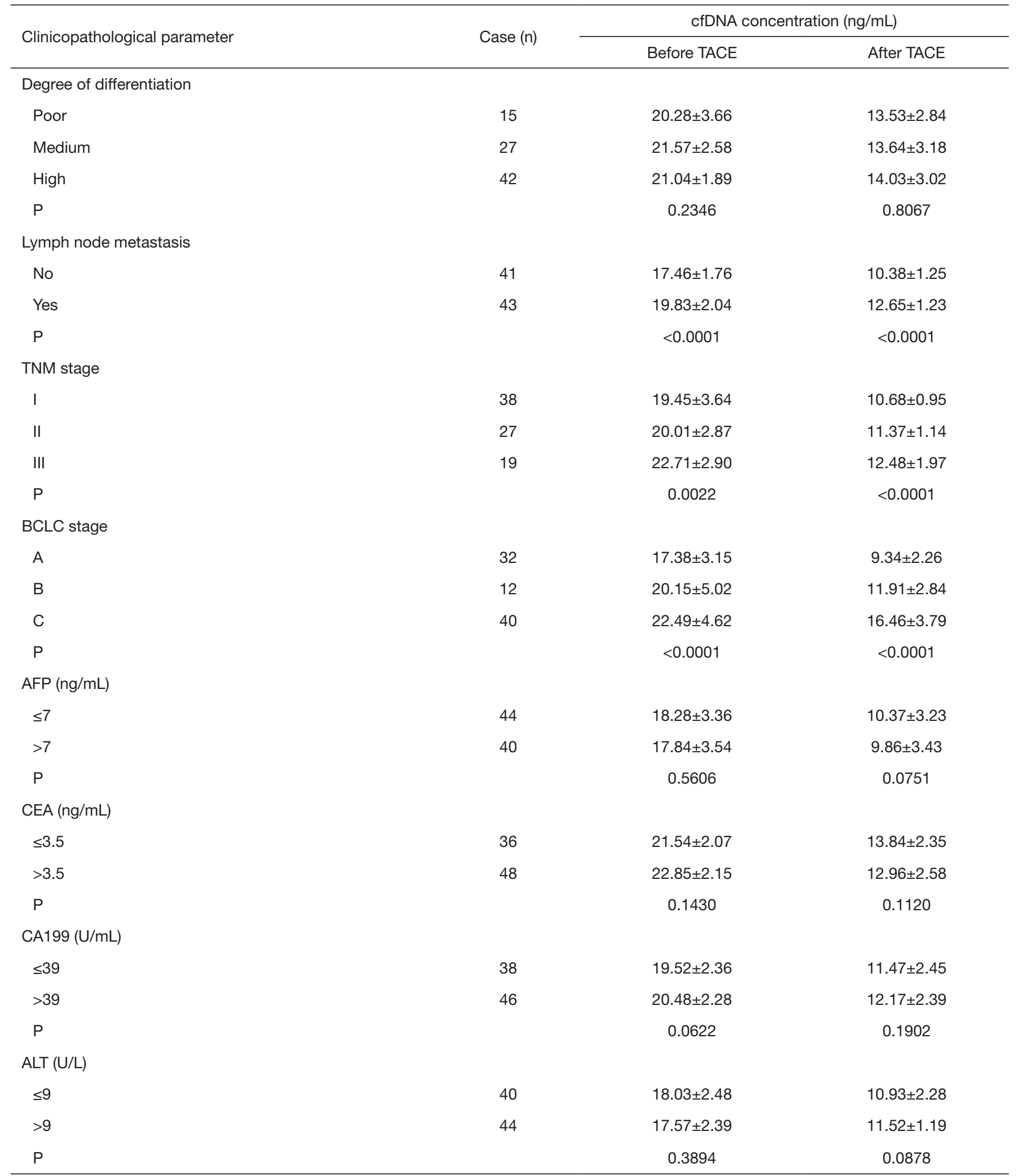

cfDNA, cell-free DNA; PHC, primary hepatocellular carcinoma; TACE, transcatheter arterial chemoembolization; AFP, alpha fetoprotein; CEA, carcinoembryonic antigen; CA199, carbohydrate antigen 199; ALT, alanine aminotransferase.

(C) Journal of Gastrointestinal Oncology. All rights reserved. J G Gastrointest Oncol 2020;11(6):1350-1363 | http://dx.doi.org/10.21037/jgo-20-509 
Table 2 Relationship between cfDNA integrity and clinical characteristics of PHC patients

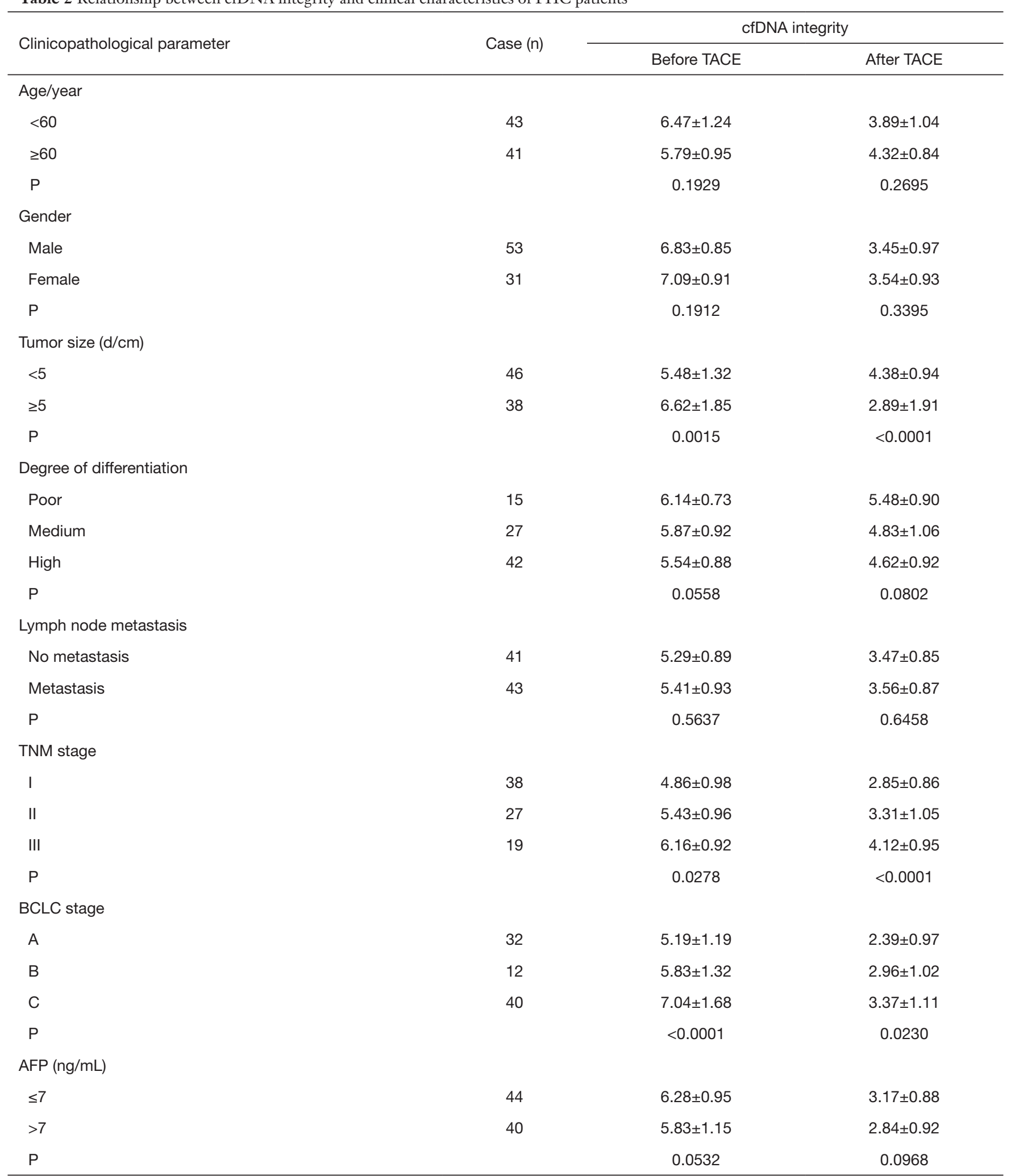

Table 2 (continued) 
Table 2 (continued)

\begin{tabular}{|c|c|c|c|}
\hline Clinicopathological parameter & Case $(n)$ & \multicolumn{2}{|c|}{ cfDNA integrity } \\
\hline \multicolumn{4}{|l|}{ CEA (ng/mL) } \\
\hline$\leq 3.5$ & 36 & $5.74 \pm 1.12$ & $2.87 \pm 0.84$ \\
\hline$>3.5$ & 48 & $6.13 \pm 0.89$ & $3.18 \pm 0.90$ \\
\hline \multicolumn{4}{|l|}{ CA199 (U/mL) } \\
\hline$\leq 39$ & 38 & $6.56 \pm .88$ & $4.55 \pm 1.92$ \\
\hline$>39$ & 46 & $7.24 \pm 1.96$ & $4.48 \pm 1.85$ \\
\hline $\mathrm{P}$ & & 0.1108 & 0.8567 \\
\hline$P$ & & 0.0723 & 0.5134 \\
\hline
\end{tabular}

cfDNA, cell-free DNA; PHC, primary hepatocellular carcinoma; TACE, transcatheter arterial chemoembolization; AFP, alpha fetoprotein; CEA, carcinoembryonic antigen; CA199, carbohydrate antigen 199; ALT, alanine aminotransferase.

patients concerning age, gender, tumor size, differentiation degree, lymph node metastasis, TNM stage, cfDNA concentration, AFP, and BCLC stage. Univariate analysis results showed that lymph node metastasis, TNM stage, cfDNA concentration, AFP, and BCLC stage were prognostic factors for PHC patients $(\mathrm{P}=0.006-0.033)$. Furthermore, COX multivariate regression analysis revealed that cfDNA concentration, TNM stage, AFP, and BCLC stage were independent risk factors for prognosis in these patients. These findings are presented in Table 3.

\section{COX multivariate analysis of factors affecting survival after TACE treatment in PHC patients}

COX multivariate regression analysis was performed with lymph node metastasis, TNM stage, cfDNA concentration, AFP, and BCLC stage from the univariate analysis results. The result shown in Table 4 revealed cfDNA concentration, TNM stage, AFP, and BCLC stage were independent risk factors for these patients' prognosis.

\section{Discussion}

A histopathological biopsy is the most valuable diagnostic method recognized internationally for PHC. However, tissue puncture is extraordinarily traumatic and is limited by tumor heterogeneity. It is unsuitable for real-time evaluation of tumor load (17). Imaging examination may not easily find small lesions, and it cannot reflect changes in tumor load with time. In recent years, the detection of tumor markers for body fluid has shown the advantages of simple operation, including reducing trauma and repeated sampling for dynamic monitoring. Since tumor cells are of a heterogeneous population, liquid biopsies supply more comprehensive molecular information than the traditional method of taking only one part of the tumor. AFP, CEA, CA199, ALT are usually used to evaluate treatment efficacy and prognosis of PHC $(18,19)$. AFP is a protein that is highly expressed in the fetus, but the expression gradually decreases or even disappears altogether after birth. However, when hepatocytes become cancerous, AFP is re-generated at elevated levels, and its content increases sharply as the disease worsens. Thus, AFP is considered a specific liver tumor marker, but its diagnostic efficacy has been found inadequate in recent years. Studies have shown $30 \%$ of patients with PHC have low expression of AFP, and in some patients with liver cirrhosis, AFP expression may be elevated for a long, but without any sign of PHC after many years $(20,21)$. Hence, as a prognostic marker of PHC, AFP has a certain proportion of false-positive and false-negative results. Although CEA, CA199, and ALT can be used as auxiliary diagnostic indicators of PHC, their sensitivities 
A

cfDNA concentration

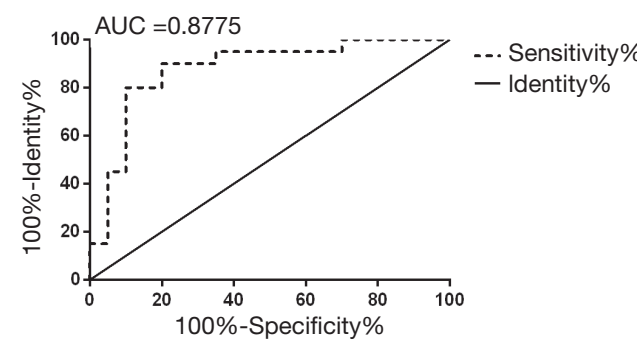

C

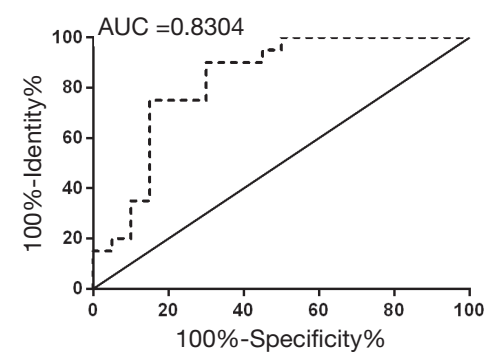

$\mathrm{E}$

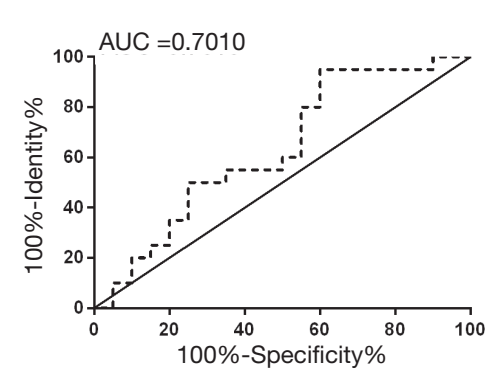

B

cfDNA integrity

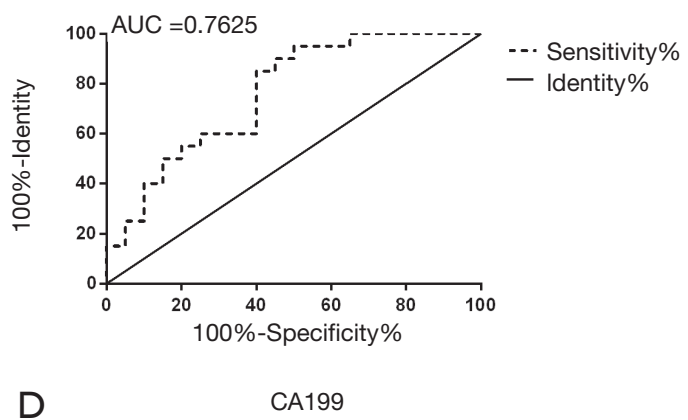

- -. Sensitivity\%
- Identity\%

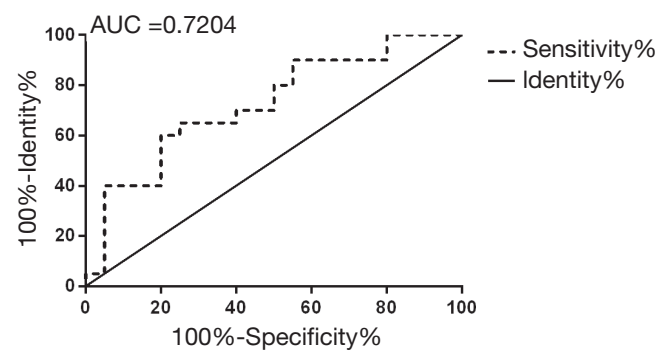

$\mathrm{F}$

CEA
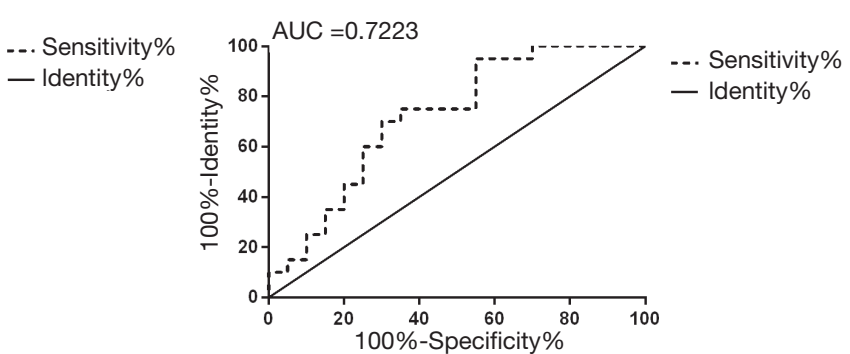

Figure 3 ROC curves of cfDNA concentration, cfDNA integrity, AFP, CEA, CA199, ALT. cfDNA, cell-free DNA; AFP, alpha fetoprotein; CEA, carcinoembryonic antigen; CA199, carbohydrate antigen 199; ALT, alanine aminotransferase.

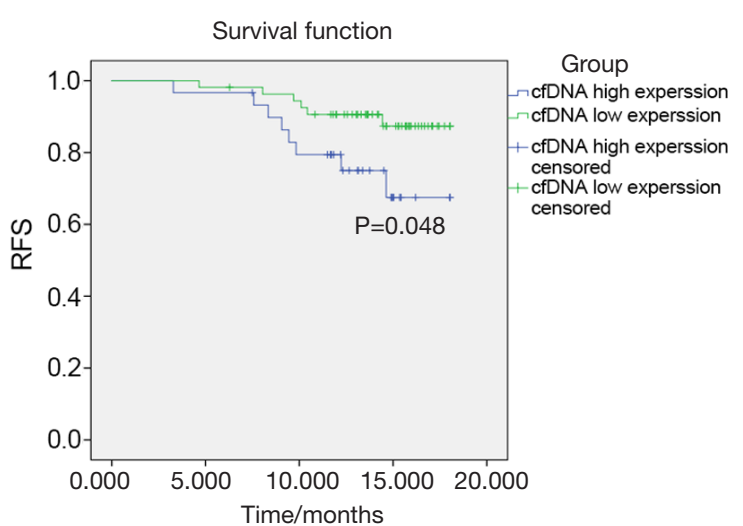

Figure 4 RFS curves of 84 PHC patients after TACE treatment. RFS, recurrence-free survival; PHC, primary hepatocellular carcinoma; TACE, transcatheter arterial chemoembolization. and specificities are not high (22). Thus, these indicators are insufficient for evaluating TACE's therapeutic effect and prognosis, and they cannot meet the requirements for the correct treatment of TACE.

This study investigated the clinical value of cfDNA in the evaluation of prognosis in patients with $\mathrm{PHC}$ after TACE treatment. The high concentration of cfDNA in most cancer cases is an independent risk factor for the disease, showing adverse consequences. In patients undergoing surgical resection of esophageal cancer, a continuous increase in cfDNA value after surgery usually shows early recurrence in follow-up (23). Recurrence or worsening of disease is detected earlier with cfDNA than with standard parameter CEA or image information, 
Table 3 Kaplan-Meier univariate survival analysis

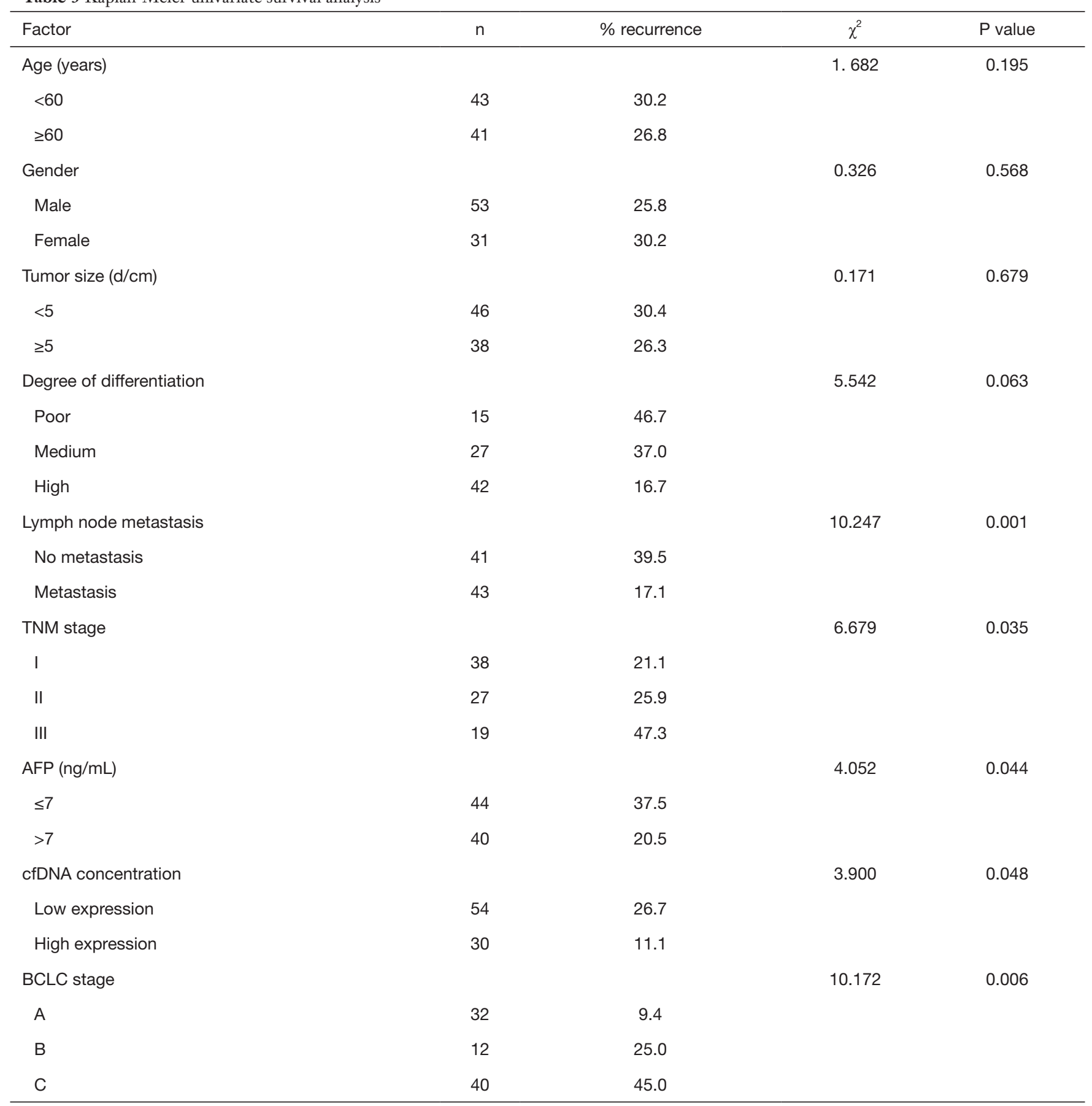

AFP, alpha fetoprotein; cfDNA, cell-free DNA.

and it has higher specificity and sensitivity. In studies on the diagnostic value of cfDNA levels in hepatocellular carcinoma, cfDNA is highly expressed in liver cancer patients and is an independent predictor of hepatocellular carcinoma (24). A comparison of area under the ROC curve revealed that the sensitivity and specificity of cfDNA concentration were higher than those of AFP. Furthermore, a study of the integrity of cfDNA in cancer patients found 
Table 4 COX multivariate regression analysis

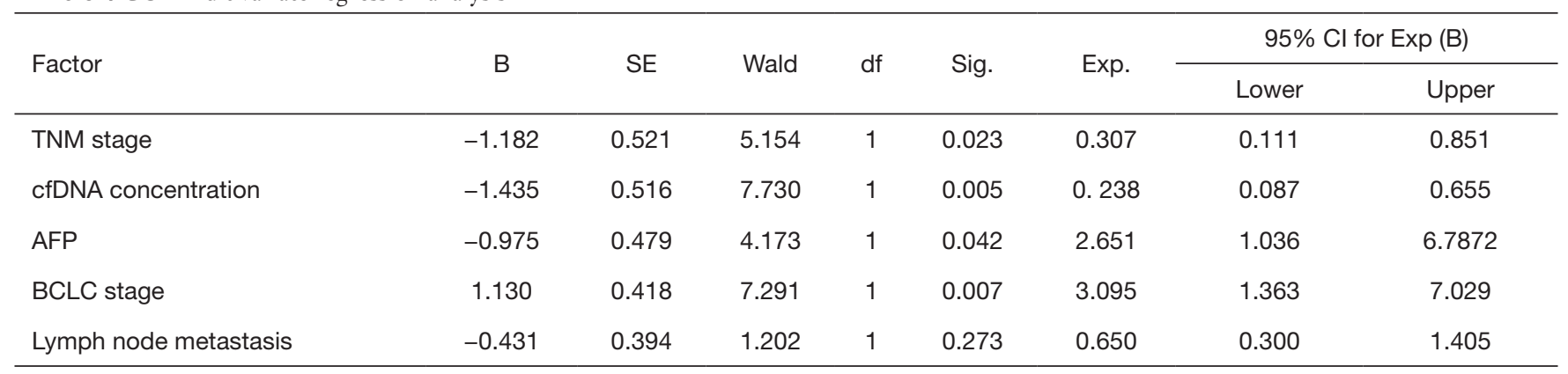

cfDNA, cell-free DNA; AFP, alpha fetoprotein.

that cfDNA integrity increased with the severity and higher stage of the disease in prostate cancer patients (25). It can be hypothesized cfDNA concentration and integrity may be sensitive indicators for evaluating prognosis in patients with PHC treated with TACE considering these studies' results.

In this study, the cfDNA concentration and integrity of PHC patients before TACE treatment were measured. The cfDNA concentration and fragment integrity of PHC patients before treatment were significantly higher than those of normal people. However, after TACE treatment, cfDNA concentration and integrity were significantly reduced. The concentration and integrity of cfDNA are correlated with treatment efficacy; the more significant the reduction in the concentration and integrity of cfDNA, the better the patient's condition. These results suggest that plasma cfDNA may be derived from liver tumor cells. After TACE treatment, tumor cell necrosis decreased, resulting in a significant reduction in the concentration and integrity of cfDNA in plasma. To further investigate the clinical reliability of cfDNA, the association of cfDNA with clinical features was analyzed. It was found cfDNA concentration and integrity were significantly associated with tumor size, TNM stage, and BCLC stage, which suggests the concentration and integrity of cfDNA reflect the growth or decline of the tumor to a certain extent. The AUC values of cfDNA concentration and integrity were greater than those of traditional tumor markers, and the sensitivity and specificity of cfDNA concentration were higher than those of the commonly used indicator AFP. Follow-up results showed that recurrence in patients with low plasma cfDNA expression after TACE treatment was significantly lower than patients with high cfDNA expression. Also, cfDNA concentration is an independent risk factor for prognosis.

In summary, plasma cfDNA concentration and integrity are highly expressed in PHC patients. However, after
TACE treatment, the levels of cfDNA and integrity changed with treatment efficacy. Therefore, cfDNA reflects the tumor burden of PHC patients, and it can be used as a reliable prognostic biomarker for PHC patients treated with TACE.

\section{Acknowledgments}

Funding: The Wuxi Municipal Health and Family Planning Commission supported this study (No. MS201735), and Foundation of Scientific Research Projects for Young Talents, Taikang Xianlin Drum Tower Hospital (No. TKKY3805).

\section{Footnote}

Reporting Checklist: The authors have completed the REMARK reporting checklist. Available at http://dx.doi. org/10.21037/jgo-20-509

Data Sharing Statement: Available at http://dx.doi. org/10.21037/jgo-20-509

Conflicts of Interest: All authors have completed the ICMJE uniform disclosure form (available at http://dx.doi. org/10.21037/jgo-20-509). The authors have no conflicts of interest to declare.

Ethical Statement: The authors are accountable for all aspects of the work in ensuring that questions related to the accuracy or integrity of any part of the work are appropriately investigated and resolved. All procedures performed in this study involving human participants were in accordance with the Declaration of Helsinki (as revised in 2013). The Ethics Committee approved the study of the 
Affiliated Hospital of the Nanjing University of Chinese Medicine. All participants supplied signed informed consent.

Open Access Statement: This is an Open Access article distributed in accordance with the Creative Commons Attribution-NonCommercial-NoDerivs 4.0 International License (CC BY-NC-ND 4.0), which permits the noncommercial replication and distribution of the article with the strict proviso that no changes or edits are made and the original work is properly cited (including links to both the formal publication through the relevant DOI and the license). See: https://creativecommons.org/licenses/by-nc-nd/4.0/.

\section{References}

1. Costentin C. Hepatocellular carcinoma surveillance. Presse Med 2017;46:381-5.

2. Mittal S, El-Serag HB. Epidemiology of hepatocellular carcinoma: consider the population. J Clin Gastroenterol 2013;47:S2-6.

3. Bteich F, Di Bisceglie AM. Current and Future Systemic Therapies for Hepatocellular Carcinoma. Gastroenterol Hepatol (N Y) 2019;15:266-72.

4. Cheung TT. Management of hepatocellular carcinoma: from bench to bedside and beyond. Transl Gastroenterol Hepatol 2019;4:54.

5. Moris D, Kostakis ID, Machairas N, et al. Comparison between liver transplantation and resection for hilar cholangiocarcinoma: A systematic review and metaanalysis. PLoS One 2019;14:e0220527.

6. Lu J, Zhong BY, Zhu HD, et al. Embolotherapy of unresectable hepatocellular carcinoma: Eastern perspective. Chin Clin Oncol 2019;8:60.

7. Cheng X, Sun P, Hu QG, et al. Transarterial (chemo) embolization for curative resection of hepatocellular carcinoma: a systematic review and meta-analyses. J Cancer Res Clin Oncol 2014;140:1159-70.

8. Sahu SK, Chawla YK, Dhiman RK, et al. Rupture of Hepatocellular Carcinoma: A Review of Literature. J Clin Exp Hepatol 2019;9:245-56.

9. Lencioni R, de Baere T, Soulen MC, et al. Lipiodol transarterial chemoembolization for hepatocellular carcinoma: A systematic review of efficacy and safety data. Hepatology 2016;64:106-16.

10. Hatamaru K, Azuma S, Akamatsu T, et al. Pulmonary embolism after arterial chemoembolization for hepatocellular carcinoma: an autopsy case report. World J
Gastroenterol 2015;21:1344-8.

11. Biolato M, Marrone G, Racco S, et al. Transarterial chemoembolization (TACE) for unresectable HCC: a new life begins? Eur Rev Med Pharmacol Sci 2010;14:356-62.

12. Zhou H, Wang XJ, Jiang X, et al. Plasma cell-free DNA chromosomal instability analysis by low-pass wholegenome sequencing to monitor breast cancer relapse. Breast Cancer Res Treat 2019;178:63.

13. Ng CKY, Di Costanzo GG, Terracciano LM, et al. Circulating Cell-Free DNA in Hepatocellular Carcinoma: Current Insights and Outlook. Front Med (Lausanne) 2018;5:78.

14. Ravaioli S. Cell-Free DNA Integrity: Applications. Methods Mol Biol 2019;1909:77-83.

15. Xu L, Huang H, Lin N. Non-invasive cell-free fetal DNA testing: a multicenter follow-up study of 31515 singleton pregnancies in southeastern China. Ultrasound Obstet Gynecol 2019;21:33.

16. Li Z, Xiao D, Li X, et al. Early recurrence detected in hepatocellular carcinoma patients after transcatheter arterial chemoembolization treatment with plasma cellfree DNA. Eur J Gastroenterol Hepatol 2019;31:885-92.

17. Balaceanu LA. Biomarkers vs. imaging in the early detection of hepatocellular carcinoma and prognosis. World J Clin Cases 2019;7:1367-82.

18. Mehta N, Dodge JL, Grab JD, et al. National Experience on Down-staging of Hepatocellular Carcinoma before Liver Transplant: Influence of Tumor Burden, AFP, and Wait Time. Hepatology 2019;2:25.

19. Montal R, Andreu-Oller C, Bassaganyas L, et al. Molecular portrait of high alpha-fetoprotein in hepatocellular carcinoma: implications for biomarker-driven clinical trials. Br J Cancer 2019;121:340-3.

20. Lee H, Yoon JH, Kim H, et al. False Positive Diagnosis of Hepatocellular Carcinoma in Liver Resection Patients. J Korean Med Sci 2017;32:315-20.

21. Gao J, Song P. Combination of triple biomarkers AFP, AFP-L3, and PIVAKII for early detection of hepatocellular carcinoma in China: Expectation. Drug Discov Ther 2017;11:168-9.

22. Edoo MIA, Chutturghoon VK, Wusu-Ansah GK, et al. Serum Biomarkers AFP, CEA and CA19-9 Combined Detection for Early Diagnosis of Hepatocellular Carcinoma. Iran J Public Health 2019;48:314-22.

23. Banki F, Yacoub WN, Hagen JA, et al. Plasma DNA is more reliable than carcinoembryonic antigen for diagnosis of recurrent esophageal cancer. J Am Coll Surg 2008;207:30-5. 
24. Yan L, Chen Y, Zhou J, et al. Diagnostic value of circulating cell-free DNA levels for hepatocellular carcinoma. Int J Infect Dis 2018;67:92-7.

25. Arko-Boham B, Aryee NA, Blay RM, et al. Circulating cell-free DNA integrity as a diagnostic and prognostic

Cite this article as: Ma K, Liu J, Wang Y, Zhong Y, Wu Z, Fan R, Guo S. Relationship between plasma cell-free DNA (cfDNA) and prognosis of TACE for primary hepatocellular carcinoma. J Gastrointest Oncol 2020;11(6):1350-1363. doi: 10.21037/jgo-20-509 marker for breast and prostate cancers. Cancer Genet 2019;235-236:65-71.

(English Language Editor: J. Chapnick) 